\title{
Hyperfractionated radiotherapy and concurrent chemotherapy in locally advanced hypopharyngeal cancers- an institutional experience
}

\author{
Thejaswini $B^{1}$, Nanda $R^{2}$, Aradhana $K^{3}$, Ashalatha ${ }^{4}$, Giri G.V ${ }^{5}$, Shamsundar 6 \\ ${ }^{1}$ Dr. Thejaswini B, Associate Professor, ${ }^{2}$ Dr. R Nanda, Associate Professor, ${ }^{3}$ Dr. Aradhana K, Associate Professor, \\ ${ }^{4}$ Dr. Ashalatha, Senior Resident, ${ }^{5}$ Dr. G.V. Giri, Professor, ${ }^{6}$ Dr. Shamsundar, Assistant Professor; all are affiliated with \\ Department of Radiation Oncology, Kidwai Memorial Institute of Oncology, Hosur Road, Bangalore.
}

Address for Correspondence: Dr. Thejaswini B, Associate Professor, Department of Radiation Oncology, Kidwai Memorial Institute of Oncology, Hosur Road, Bangalore. Email id: thejaswiniumesh@gmail.com

\begin{abstract}
Aim: To determine whether hyperfractionated radiotherapy with concurrent cisplatin improves outcome in advanced hypopharyngeal cancers. Methodology: From 2012 to 2014, twenty three patients diagnosed with squamous cell carcinoma of hypopharynx (Stg III-7, Stg IVA-14, and Stg IVB-2) were assigned to receive total dose of 75.9 Gy in 66 fractions@1.15 Gy/fraction, twice daily with inter-fraction interval of 6 hours, 5 days/week, over 45 days. Spinal cord shielding was done at 50.6 Gy in 44 fractions. Concurrent chemotherapy was delivered with cisplatin $40 \mathrm{mg} / \mathrm{m} 2 \mathrm{once}$ weekly. The tumor response was assessed at 6 weeks and 3 months after the completion of treatment. Results: All the recruited 23 patients completed the planned treatment. The complete response at 6 weeks and 3 months post treatment for primary and node was observed in $16 / 23$ and 18/23, 13/23 and 16/23 patients respectively. The grade I and II toxicities encountered during the treatment were mucositis (16 patients), radiation dermatitis (20 patients), neutropenia (9 patients), and leucopenia (18 patients). Grade 3 reactions seen were mucositis (6 patients), neutropenia ( 3 patients), leucopenia ( 2 patients), skin ( 2 patients) and none of the patients had grade IV reactions. The nutrition was maintained with feeding nasogastric tubes and parentral infusion though out the treatment. The median follow up was 18 months with a median overall survival of 24 months and median disease free survival was 18 months without any statistical significance. Conclusion: Hyperfractionated radiotherapy combined with concurrent weekly cisplatin treatment for locally advanced hypopharyngeal cancers can be considered with rigorous monitoring and management of the toxicities.
\end{abstract}

Key words: Concurrent Chemoradiation; Head and Neck Cancers; Hyperfractionated Radiotherapy; Locally Advanced Hypopharyngeal Cancer; Weekly Cisplatin.

\section{Introduction}

Head and neck cancers constitute about $30.6 \%$ and $14.4 \%$ of all cancers in males and females respectively. Hypopharynx constitutes $7 \%$ in males and $1.5 \%$ in females [1]. Majority of the patients present with locally advanced disease for which the recommended treatment is combined modality. At present the standard of care in patients with locally advanced HNSCC are; surgery followed by postoperative radiotherapy with or without chemotherapy, concurrent chemoradiation or altered fractionation with surgical salvage [2]. Altered fractionation regimens have been tested with advantages and disadvantages. In hyperfractionation,

Manuscript received $6^{\text {th }}$ May 2016

Reviewed: $20^{\text {th }}$ May 2016

Author Corrected: $4^{\text {th }}$ June 2016

Accepted for Publication $18^{\text {th }}$ June 2016 the total dose is increased, the size of dose per fraction is significantly reduced, the number of fractions is increased, and overall treatment time is relatively unchanged. The basic rationale of hyperfractionated radiotherapy is that the use of small dose fractions allows higher total doses to be administered within the tolerance of late-responding normal tissues, and this translates into a higher biologically effective dose to the tumor. Other rationales for hyperfractionation are radiosensitization through redistribution and lesser dependence on oxygen effect. The greater the number of dose fractions, the greater the chance that cells would be in a more radiosensitive phase at the time of the next fraction [3]. 
Radiotherapy with concurrent chemotherapy is another therapeutic option in locally advanced head and neck squamous cell carcinoma. The rationale of this combination is that chemotherapy would improve locoregional tumor control, while controlling the possibility of distant metastasis. The efficacy of which depends on the drugs given in combination or as single. The drugs that are commonly used are 5FU, cisplatin, carboplatin and mitomycin. Among the drugs used concurrently with radiotherapy, cisplatin has been frequently used because the toxicities do not overlap with that of radiotherapy [4].

The study was conducted to exploit the advantages of both hyperfractionated radiotherapy and concurrent chemotherapy.

\section{Materials and Methods}

Between 2012-14, twenty-three patients of locally advanced hypopharyngeal squamous cell carcinoma (stage III and IV) with ECOG status of 0 or 1 , and no associated comorbid conditions were included in the study. The patient characteristics are shown in Table 1.

Table-1: Patient Characteristics.

\begin{tabular}{|l|l|l|l|}
\hline \multicolumn{2}{|l|}{ Patient Characteristics } & No. & $\%$ \\
\hline \multirow{3}{*}{ Age } & $<50$ & 7 & 30.4 \\
\cline { 2 - 4 } & $>/=50$ & 16 & 69.6 \\
\hline \multirow{3}{*}{ Tobacco consumption } & Yes & 21 & 91.3 \\
\cline { 2 - 4 } & No & 2 & 8.7 \\
\hline \multirow{3}{*}{ Chemotherapy dose } & $</=200 \mathrm{mg}$ & 9 & 39.1 \\
\cline { 2 - 4 } & $>200 \mathrm{mg}$ & 14 & 60.9 \\
\hline \multirow{3}{*}{ Chemotherapy no. of cycles } & $<4$ & 8 & 34.8 \\
\cline { 2 - 4 } & $04-J u n$ & 15 & 65.2 \\
\hline \multirow{3}{*}{ Stage } & III & 7 & 30.4 \\
\cline { 2 - 4 } & IVA & 14 & 60.9 \\
\cline { 2 - 4 } & IVB & 2 & 8.7 \\
\hline
\end{tabular}

The pretreatment evaluation was done with clinical examination, blood investigations including haemogram, renal and hepatic function tests and dental prophylaxis. Pathological confirmation was done with biopsy of primary and FNAC of the neck nodes. Radiological investigations such as chest x-ray, ultrasound abdomen, computed tomographic study of head and neck was mandatory. Pre chemotherapy investigations like audiometry, glomerular filtration rate and ECHO cardiogram was also done in all patients. Special informed consent, Institutional scientific review board and ethical committee clearance was obtained as matter of Institute policy.

Treatment Protocol: All patients were treated on telecobalt at $80 \mathrm{~cm} \mathrm{SSD} \mathrm{with} \mathrm{hyperfractionation,} \mathrm{i.e.,} \mathrm{two} \mathrm{daily}$ fractions of $1.15 \mathrm{~Gy}$ with inter-fraction interval of six hours to a total dose of $75.9 \mathrm{~Gy}$ in 66 fractions (2Gy equivalent $70.53 \mathrm{~Gy}$ ) in 45 treatment days over 6.5 weeks from Monday to Friday. The primary and neck nodes were treated using AP-PA fields to a dose of 50.6 Gy in 44 fractions (2Gy equivalent $47.02 \mathrm{~Gy}$ ). This was followed by bilateral parallel opposing fields sparing the spinal cord to a dose of $25.3 \mathrm{~Gy}$ in 22 fractions (2Gy equivalent 23.51Gy). Concurrent cisplatin was given $(40 \mathrm{mg} / \mathrm{m} 2)$ weekly once, before the second fraction of radiotherapy.

Treatment Monitoring: All patients were admitted and monitored during treatment. Hydration, protein, caloric intake and oral hygiene were adequately maintained. Haemogram and biochemical investigations were done weekly before giving chemotherapy. Weekly assessment was done for weight loss, primary and nodal response. Depending on the chemotherapy related toxicities cisplatin was discontinued but radiotherapy was continued as planned. Both the primary and nodal response was assessed at 6 weeks and 3 months after the completion of treatment. 
The tumor response was assessed as per the RECIST Criteria (2009, v1.1). Acute mucosal and skin toxicity was assessed as per the RTOG Acute Radiation Morbidity Scoring system. Chemotherapy induced toxicity like renal and hematological toxicities were assessed as per the Common Terminology Criteria for Adverse Events version 4.3.

The first and second follow-up was done at 6 weeks and 3 months after the completion of the treatment. Clinical examination and a CT scan were used in the evaluation of response.

Statistical Methods: Descriptive statistical analysis is done in this study. Results on categorical measurements are presented in number $(\%)$. The relative percentage was used to study the toxicities. The overall and disease free survival was calculated using $\mathrm{R}$ software.

\section{Results}

All patients completed treatment within 45 to 51 days. The toxicities encountered, during and after the treatment are as shown in Table 2. Out of 23 patients accrued 12 patients had anemia before or during treatment and they were transfused with 3 to 5 pints of blood. Either due to mucositis and/or dysphagia, swallowing was impaired in 21 patients. Nasogastric tube was inserted during 1st week of radiation to maintain nutrition. Despite this, nutrition was not adequate leading to significant weight loss (median-3kgs) in 21 patients. Hence, parenteral nutrition [combination of carbohydrates, proteins and lipids] was also given.

Table- 2: Toxicities during and after the treatment

\begin{tabular}{|l|l|l|l|l|l|l|}
\hline Reactions & Gr 0 & $\%$ & Gr I-II & $\%$ & Gr III & $\%$ \\
\hline Leukopenia & 3 & 12.5 & 18 & 75 & 2 & 8.3 \\
\hline Neutropenia & 11 & 45.8 & 9 & 37.5 & 3 & 12.5 \\
\hline Alopecia & 21 & 87.5 & 2 & 8.3 & 0 & 0 \\
\hline Skin & 0 & 0 & 21 & 87.5 & 2 & 8.3 \\
\hline Mucositis & 0 & 0 & 17 & 73.9 & 6 & 26.1 \\
\hline Dysphagia & 12 & 52.2 & 9 & 39.1 & 2 & 8.7 \\
\hline Laryngeal Edema & 12 & 52.2 & 11 & 47.8 & 0 & 0 \\
\hline UGI & 4 & 17.4 & 17 & 73.9 & 2 & 8.7 \\
\hline
\end{tabular}

In majority of patients mucositis appeared from end of second week during treatment and resolved by 4 to 8 weeks posttherapy. Epilation (8 patients) and patchy dry desquamation appeared from third week onwards. Xerostomia (7 patients) appeared after 12th week and persisted till about 14 to 28 weeks post radiotherapy. Laryngitis (11 patients) appeared from 4 th week onwards.

About 8 patients have received only 3 cycles of chemotherapy [ $<200$ mgtotal dose] due to leukopenia and neutropenia [grade 2-3]. Only two patients had thrombocytopenia. However, none of the patients had renal toxicity.

Table-3: Treatment response assessment.

\begin{tabular}{|l|l|l|l|l|l|l|l|l|}
\hline \multirow{3}{*}{ Response } & \multicolumn{3}{l}{ Response at 6 Weeks } & \multicolumn{3}{l|}{ Response at 3 Months } \\
\cline { 2 - 10 } & \multicolumn{2}{|l|}{ Primary } & \multicolumn{2}{l|}{ Node } & \multicolumn{2}{l|}{ Primary } & \multicolumn{2}{l|}{ Node } \\
\cline { 2 - 10 } & $\#$ & $\%$ & $\#$ & $\%$ & $\#$ & $\%$ & $\#$ & $\%$ \\
\hline CR & 16 & 69.6 & 13 & 56.5 & 18 & 78.3 & 16 & 69.6 \\
\hline PR & 5 & 21.7 & 5 & 21.7 & 2 & 8.7 & 1 & 4.3 \\
\hline PD & 2 & 8.7 & 2 & 8.7 & 3 & 13.0 & 3 & 13.0 \\
\hline
\end{tabular}




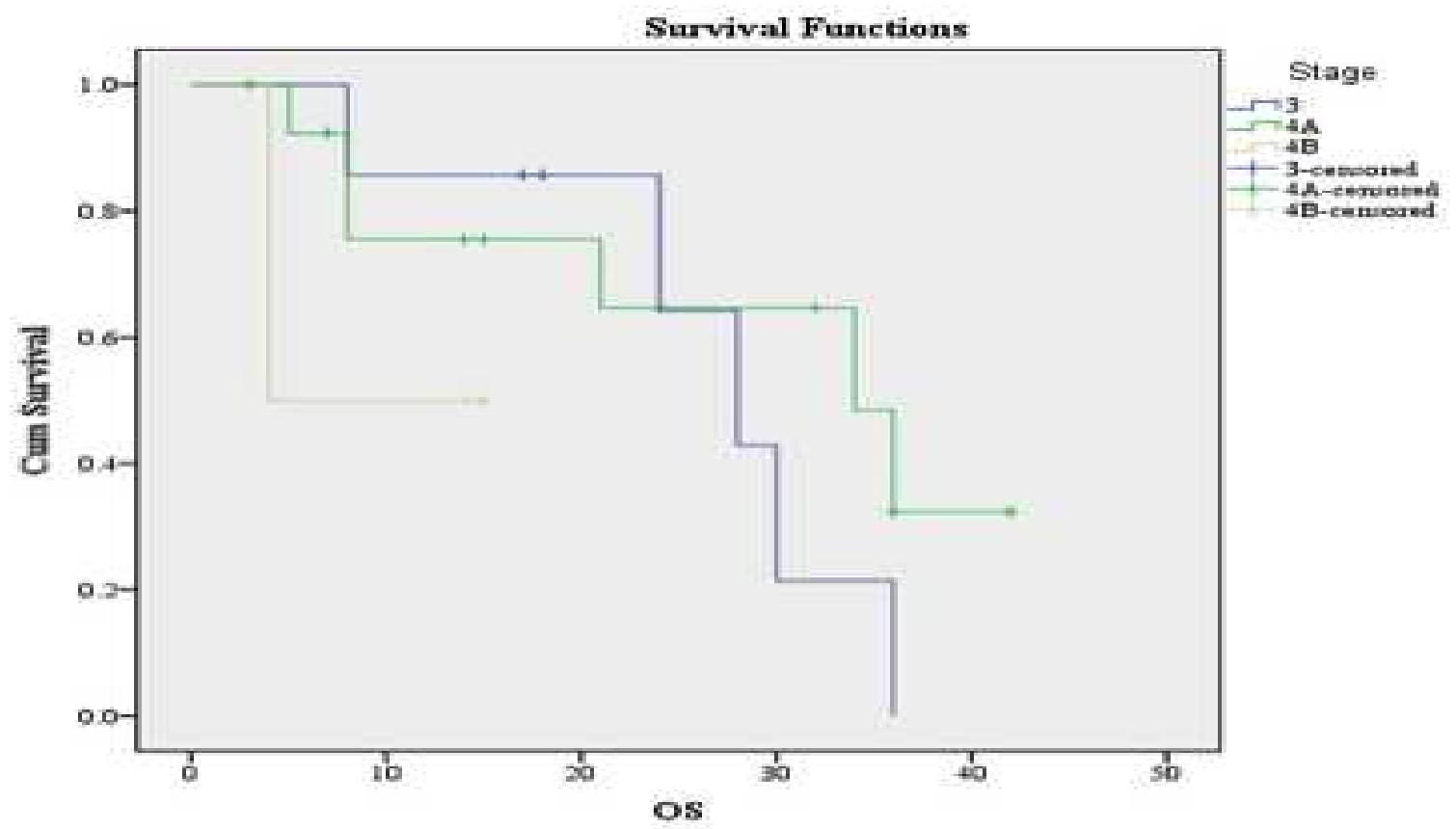

Fig 1: Overall survival

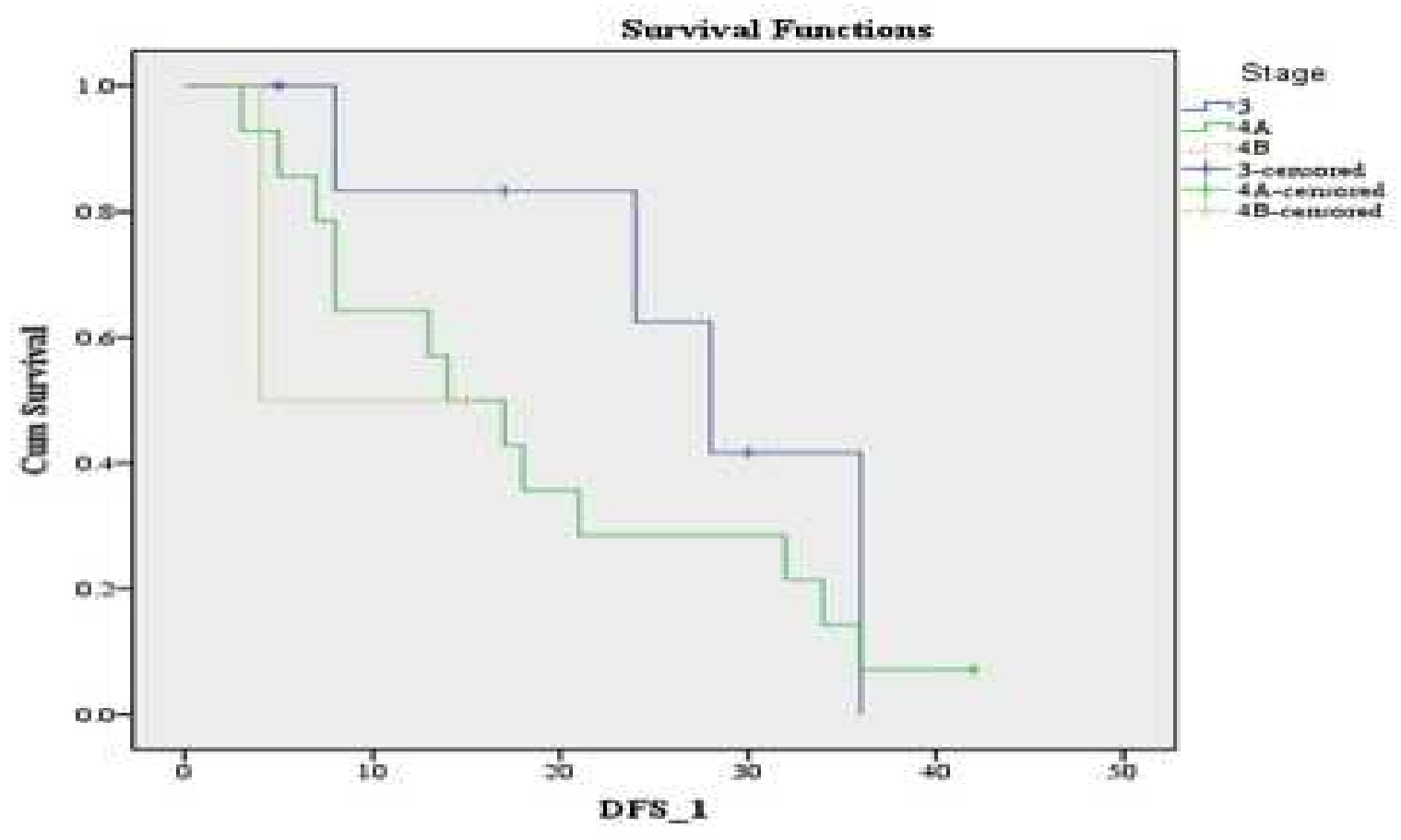

Fig 2: Disease free survival

The post treatment response at 6 weeks and 3 months have shown in Table 3. Median follow up period was 18 months. Median overall survival was 24 months and median disease free survival was 18 months (Fig 1 \& 2). Disease free survival was poor (10 months) in stage IV. Although complete response rate of $68 \%$ to $75 \%$ at 3 months was achieved, 4 patients died due to pneumonitis [2pts], second malignancy [1pt] and intestinal perforation [1pt]. The numbers of nontumour related deaths have obscured the survival advantage. 8 patients died due to residual/progressive disease. Salvage surgery was not done in two patients as these patients refused further treatment. 


\section{Discussion}

Hyperfractionated radiotherapy and concurrent chemoradiotherapy has consistently shown superiority over conventional fractionation regimes in advanced head and neck cancers. The results of six phase III trials, testing the efficacy of such combinations against radiation alone have been reported $[6,7,8,9,10,11,12]$.

The radiation regimens used were hyper-fractionation in four trials $[6,7,9,10]$. Most trials show that combinations of altered fractionation regimens with chemotherapy achieve better local control. Few trials showed improved overall survival compared with standard or altered fractionation alone.

In our study $80 \%$ of patients completed the intended treatment protocol of chemoradiation in 45 days. As per Olfred Hansen prolongation of the overall treatment time lead to reduced loco-regional control [21]. Withers, Bentzen and Thames et al showed that if the duration of radiotherapy prolonged beyond three to four weeks, the effect of each additional day is equivalent to a loss of dose effectiveness of 0.48 Gy $[13,14,15]$.

Nine patients (40\%) completed four cycles of CT and six patients $(26 \%)$ received more than four cycles of CT. In MACH-NC meta-analysis an addition of chemotherapy to radiotherapy has shown a small but significant survival advantage in favour of chemotherapy $[19,20]$.

The significant toxicity in our study was mucositis $26 \%$ of the patients had grade III, $74 \%$ had grade I-II and none had grade IV. The incidence of grade $3+$ mucositis is less (55\% vs. $65-75 \%)$ as compared to Brizel and other studies with similar schedules $[6,7,8,12]$. Grade II mucositis was seen during the third week, while grade III mucositis was seen during the fourth week of treatment. All patients responded to the conservative management.

Mucositis compromised ability to maintain nutrition in patients, on an average, having lost $6.4 \%$ of baseline weight, comparable to study by Brizel et al which reported $10 \%$ mean weight loss [6].

Eighty seven percent of the patients had grade I-II and $8.3 \%$ of the patients had grade III skin reaction. As per Fernando la Vega incidence of Grade III cutaneous toxicity was $23 \% 5$ whereas Azza Abd El-Naby reported Grade III skin reaction $3.8 \%$ and Grade II as $50 \%[5,16]$.
Jeremic et al reported that Grade III leucopenia was 12 $\%$ and $5 \%$ of patients had renal toxicity [9]. Dobrowsky and Naude reported that grade 3-4 hematological toxicity was $18 \%$ (cisplatin was given $100 \mathrm{mg} / \mathrm{m} 2$ ) [8]. In our study, Grade III leucopenia was $8.3 \%$ and Grade I-II was $75 \%$ whereas Grade III neutropenia was $12.5 \%$ and Grade I-II was 37.5\%. No renal toxicity was observed in our study.

In a study by Azza Abd El-Naby Grade III dysphagia was reported as $27 \%$, Grade II as $65 \%$ and Grade I was $4 \%$, whereas $43 \%$ patients had persistent dysphagia for both solids and semi solids and $12 \%$ patients had persistent dysphagia for liquids [16]. In our study $47.8 \%$ had Grade I-II and $8.7 \%$ of patients had Grade III dysphagia.

Both meta-analyses and randomized trials have established the role of concurrent chemotherapy in improving outcomes in head-and-neck cancers [17]. The recent update from Denis et al shows an improvement in overall survival and locoregional control at 5 years using combined modality therapy for advanced-stage head and neck cancers [18]. It follows that the combined use of altered fractionation and concurrent chemotherapy in our study is likely a contributing factor to the observed rates of initial tumor response and acute toxicities.

The tumor response assessed in our study was $69 \%$ and $78 \%$ at 6 weeks and 3 months post treatment which was comparable with the Brizel study, wherein $88 \%$ of patients achieved complete response with median follow up of 41 months [6]. Similarly, Fernando la Vegaet al showed 63\% complete response, 34\% partial response and 3\% stable disease [5]. Jeremic et al reported $65 \%$ complete response with median follow up of 79 months in his study [9]. All these studies reported that the whole benefit of hyperfractionation came from improved tumor control, which further improved with chemotherapy. Budach et al showed substantial prolongation of median survival of 14.2 months $(\mathrm{p}<0.001)$ for hyperfractionated radiotherapy alone compared with conventional fractionation. They concluded that any altered fractionation in combination with concurrent chemotherapy resulted in an overall survival benefit of 12 months compared with altered fractionation alone [11]. In contrast, our study showed that the overall survival and the disease free survival was not statistically significant ( $p$ value 0.243 and 0.167 respectively). 


\section{Conclusion}

This prospective study showed that hyperfractionated radiotherapy with concurrent weekly cisplatin is feasible without significant treatment interruptions and resulted in $70 \%$ of complete response in locally advanced hypopharyngeal cancers. The compliance to radiotherapy was excellent as the patients were admitted. The improved response was associated with increase in acute toxicity leading to impaired nutrition and weight loss. But this was appropriately managed with nasogastric tube feeding and parentral nutrition. However, due to the increased treatment related toxicities encountered, further accrual of patients was stopped and the study was terminated.

Acknowledgement: Dr. Nanda. R and Dr. Aradhana. $\mathrm{K}$, have contributed equally for this study. I thank all the people associated with this study directly or indirectly. I also thank Dr. Vijay. C. R, Assistant professor in statistics, Kidwai Memorial Institute of Oncology for the valuable guidance and statistical analysis.

Funding: Nil, Conflict of interest: None initiated.

Permission from IRB: Yes

\section{References}

1. Hospital Based Cancer Registry: Kidwai Memorial Institute of Oncology, Bangalore: Annual Report 2012; Head and Neck Cancers: 27-27.

2. National Comprehensive Cancer Network. Practice Guidelines: Head and Neck Cancer, version 1.2009. http/www.nccn.org/physician-gls/PDF/head-and-neckpdf.

3. Halperin EC, Perez CA, Brady LW. Principles and Practice of Radiation Oncology. 6th Edition. Lippincott Williams and Wilkins Publishers; 2008.

4. Huguenin P, Beer KT, Allal A et al. Concomitant cisplatin significantly improves locoregional control in advanced head and neck cancers treated with hyperfractionated radiotherapy. J Clin Oncol. 2004 Dec 1;22(23):4665-73. Epub 2004 Nov 8.doi:10. 1200/JCO. 2004.12.193.

5. Fernando Arias de la Vega, Miguel A Dominguez et al. Hyperfractionated radiation therapy and cisplatin for locally advanced head and neck cancer. A non randomized comparison of quality of life between two consecutive treatment protocols at a single institution. Cancer therapy.2007; Vol 5: 161-8.

6. Brizel DM, Albers ME, Fisher SR, Scher RL, Richtsmeier WJ, Hars V, George SL, Huang AT, Prosnitz LR. Hyperfractionated irradiation with or without concurrent chemotherapy for locally advanced head and neck cancer. N Engl J Med. 1998 Jun 18;338 (25):1798-804.

7. Budach V, Stuschke M, Budach W, Baumann W, Geismar D, Grabenbauer G et al. Hyperfractionated accelerated chemoradiation with concurrent fluorouracil-mitomycin is more effective than doseescalated hyperfractionated accelerated radiation therapy alone in locally advanced head and neck cancer: final results of the radiotherapy cooperative clinical trials group of the German Cancer Society 95-06 prospective randomized trial. J. Clin. Oncol. $2005 \mathrm{Feb}$ 20; 23(6):1125-35. doi: 10.1200/JCO.2005.07.010.

8. Dobrowsky W, Naudé J. Continuous hyperfractionated accelerated radiotherapy with/ without mitomycin $\mathrm{C}$ in head and neck cancers. Radiother Oncol. 2000 Nov;57(2):119-24.

9. Jeremic B, Shibamoto Y, Milicic B, Nikolic N, Dagovic A, Aleksandrovic J, Vaskovic Z, Tadic L. Hyperfractionated radiation therapy with or without concurrent low-dose daily cisplatin in locally advanced squamous cell carcinoma of the head and neck: a prospective randomized trial. J Clin Oncol. 2000 Apr;18(7):1458-64.

10. Wendt TG, Grabenbauer GG, Rodel CM, Thiel HG, Aydin H, Rohloff $\mathrm{R}$ et al. Simultaneous radiochemotherapy versus radiotherapy alone in advanced head and neck cancer: a randomized multicenter study. J Clin Oncol. 1998 Apr 16;16(4): $1318-24$.

11.Budach W, Hehr T, Budach V, Belka C, Dietz K. A meta-analysis of hyperfractionated and accelerated radiotherapy and combined chemotherapy and radiotherapy regimens in unresected locally advanced squamous cell carcinoma of the head and neck. BMC Cancer. 2006 Jan 31; 6: 28.doi:10.1186/1471-2407-6-28

12. Staar S, Rudat V, Stuetzer H, Dietz A, Volling P, Schroeder $\mathrm{M}$ et al. Intensified hyperfractionated accelerated radiotherapy limits the additional benefit of 
simultaneous chemotherapy: Results of a multicentric randomized German trial in advanced head-and-neck cancer. Int J Radiat Oncol Biol Phys. 2001 Aug 1; 50 (5): 1161-71.

13. Withers HR, Taylor JM, Maciejewski B. The hazard of accelerated tumor clonogen repopulation during radiotherapy. Acta Oncol. 1988;27(2):131-46.

14. Bentzen SM, Thames HD. Clinical evidence for tumor clonogen regeneration: interpretations of the data. Radiother Oncol. 1991 Nov; 22(3):161-6.

15. Fowler JF, Lindstrom MJ. Loss of local control with prolongation in radiotherapy. Int $\mathbf{J}$ Radiat Oncol Biol Phys. 1992;23(2):457-67.

16. Azza Abd El-Naby, Aly Tawfek et al. Conventional versus Hyperfractionated radiotherapy in locally advanced head and neck cancer. Cancer Therapy. 2008; 6(2):673-82.

17. Pignon JP, le Maître A, Maillard E, Bourhis J; MACH-NC Collaborative Group. Meta-analysis of chemotherapy in head and neck cancer (MACH-NC): an update on 93 randomised trials and 17,346 patients. Radiother Oncol. 2009 Jul;92(1):4-14. doi: 10.1016/j. radonc.2009.04.014. Epub 2009 May 14.
18. Denis F, Garaud P, Bardet E, Alfonsi M, Sire C, Germain $\mathrm{T}$ et al. Final results of the 94-01 French Head and Neck Oncology and Radiotherapy Group randomized trial comparing radiotherapy alone with concomitant radiochemotherapy in advanced-stage oropharynx carcinoma. J Clin Oncol. 2004 Jan 1; 22(1) :69-76.doi:10.1200/JCO.2004.08.021.Epub 2003 Dec 2.

19. Pignon JP, Bourhis J et al. Chemotherapy added to loco-regional treatment for head and nech squamous cell carcinoma: three meta-analysis of updated individual data. MACH-NC collaborative group. MetaAnalysis of chemotherapy on head and neck cancers. Lancet. 2000 Mar 18; 355 (9208):949-55.

20. Bourhis J, Amand C et al. On behalf of MACH-NC collaborative group: Update of MACH-NC (MetaAnalysis of chemotherapy in head and neck cancer) database focused on concomitant chemoradiotherapy. J Clin Oncol. 2004; ASCO annual meeting proceeding (Post meeting edition): 22(No 14S (Jul 15 supplement)): 5505. ISSN 7032-183X.

21. Hansen O, Overgaard J, Sand Hansen H, et al. Importance of overall treatment time for the outcome of radiotherapy of advanced head and neck carcinoma: dependency on tumor differentiation. Radiother Oncol. 1997 Apr; 43(1):47-51.DOI: http://dx.doi.org/10. 1016/ S0167-8140(97)01904-X.

\section{How to cite this article?}

Thejaswini B, Nanda R, Aradhana K, Ashalatha, Giri G.V, Shamsundar. Hyperfractionated radiotherapy and concurrent chemotherapy in locally advanced hypopharyngeal cancers- an institutional experience. Int J Med Res Rev 2016;4 (6):1017-1023.doi: 10.17511/ijmrr.2016.i06.26. 